\title{
Indirect Boundary Stabilization of a System of Schrödinger Equations with Variable Coefficients
}

Ilhem Hamchi and Salah Eddine Rebiai

\begin{abstract}
We consider a coupled system of two complex Schrödinger equations with variable coefficients. The boundary feedback appears only in one of the equations. The aim of this paper is to prove that we can apply the Riemann geometric approach developed to study the problems of direct stabilization for real hyperbolic equations (see [22]) and show that the sufficiently smooth solutions decays polynomially at infinity, by adapting the ideas of Alabau in [2] used to obtain indirect stabilization results for a system of two coupled real wave equations with constant coefficients.
\end{abstract}

Mathematics Subject Classification (2000). 93D15, 35Q40, 42B15.

Keywords. Riemannian geometry, Indirect damping, Multiplier method.

\section{Introduction}

Let $\Omega \subset \mathbb{R}^{n}, n \in \mathbb{N}^{*}$, be an open bounded domain with $C^{2}$ boundary $\Gamma=\Gamma_{0} \cup \Gamma_{1}$ such that $\Gamma_{0} \neq \emptyset$ and $\overline{\Gamma_{0}} \cap \overline{\Gamma_{1}}=\emptyset$.

$$
\mathcal{A} y=-\sum_{i, j=1}^{n} \frac{\partial}{\partial x_{i}}\left(a_{i j}(x) \frac{\partial y}{\partial x_{j}}\right)
$$

is a second order differential operator with real coefficients $a_{i j}=a_{j i}$ of class $C^{1}$ and satisfies the uniform ellipticity condition

$$
\sum_{i, j=1}^{n} a_{i j}(x) \zeta_{i} \zeta_{j} \geq a_{0} \sum_{i=1}^{n} \zeta_{i}^{2} \quad \text { for all } \quad x \in \Omega \quad \text { and } \quad \zeta=\left(\zeta_{1}, \zeta_{2}, \ldots, \zeta_{n}\right) \in \mathbb{R}^{n},
$$

for some positive constant $a_{0}>0 . v=\left(v_{1}, \ldots, v_{n}\right)$ is the outward unit normal to $\Gamma, \frac{\partial y}{\partial v_{\mathcal{A}}}=\sum_{i, j=1}^{n} a_{i j}(x) \frac{\partial y}{\partial x_{j}} v_{i}$ denotes the co-normal derivative with respect to $\mathcal{A}$. 
$a$ and $b$ are two functions in $L^{\infty}(\bar{\Omega})$ such that for some constants $a_{*}, b_{*}>0$, we have

$$
a_{*} \leq a(x) \text { for all } x \in \Omega \text { and } b_{*} \leq \operatorname{Re}(b(x))=b(x) \text { for all } x \in \Gamma .
$$

Given $T>0$, let $Q=\Omega \times] 0, T[, \Sigma=\Gamma \times] 0, T\left[\right.$ and $\left.\Sigma_{l}=\Gamma_{l} \times\right] 0, T[(l=0,1)$. In $\Omega$, we consider the following coupled complex valued Schrödinger equations with variable coefficients

$$
\begin{aligned}
& \mathfrak{i} y_{t}+\mathcal{A} y+a z=0 \quad \text { in } \quad Q, \\
& \mathfrak{i} z_{t}+\mathcal{A} z+a y=0 \quad \text { in } Q, \\
& y=0 \quad \text { on } \Sigma_{0}, \quad \frac{\partial y}{\partial v_{\mathcal{A}}}+b y_{t}=0 \quad \text { on } \Sigma_{1} \text { and } z=0 \text { on } \Sigma . \\
& y(0)=y_{0} \text { and } z(0)=z_{0} \text { in } \Omega .
\end{aligned}
$$

This type of system describes a particle in a box with two levels internal and subject to a wave resonant laser with the transition between the two energy levels [20].

For the system (1)-(4), we can remark that a boundary feedback is acting on one end only (no damping acting on $z$ on $\Sigma_{1}$ ).

The problem of proving exact controllability and uniform decay rates for solutions of one Schrödinger equation with constant coefficients has been treated by several authors, we can mention $[17,18]$. Recently, Riemann geometric methods have emerged as a powerful tool to obtain continuous observability inequalities and direct stabilization for various classes of PDEs with variable coefficients, see for instance $[12,13,15,19,22,24-26]$. Another question was considered in the literature: the problem of indirect stabilization, this problem was first studied by Russell [21] who introduced the terminology of indirect damping, since the first equation can be regarded as a stabilizer for the second one. Recently, Aassila [1] proved, using spectral theory, that the solution of the following coupled wave equations

$$
\begin{aligned}
& y_{t t}-\Delta y+\lambda(y-z)=0 \text { in } Q, \\
& z_{t t}-\Delta z+\lambda(z-y)=0 \text { in } Q, \\
& y=z=0 \text { on } \Sigma_{0}, \\
& \frac{\partial y}{\partial v}+\mu y+y_{t}=0 \text { and } z=0 \text { on } \Sigma_{1}, \\
& y(0)=y_{0}, \quad z(0)=z_{0} \text { and } y_{t}(0)=y_{1}, z_{t}(0)=z_{1} \text { in } \Omega
\end{aligned}
$$

is strongly stable for arbitrary $n$ and is not uniformly exponentially stable when $\Omega$ is an interval $(n=1)$. Alabau [2] studied the indirect boundary stabilization of coupled real hyperbolic systems. She has proved that the feedback of the first equation is sufficient to stabilize polynomially the total system. So, she has established a polynomial decay lemma for a nonincreasing functional satisfying a generalized integral inequality. In [3], the authors have studied the problem of indirect stabilization of two coupled second order evolution equations via the zero order terms 
by a feedback acting in the whole domain. These results have been extended, using the piecewise multiplier, to several cases: wave-wave, Petrowsky-Petrowsky coupling, for locally distributed action [7]. We note that, in all these works, the coupling coefficient is considered as a positive constant which is sufficiently small.

The goal of this paper is to prove that we can apply the Riemann geometric approach to the coupled complex Schrödinger equations with variables coefficients (here we construct a suitable Riemannian metric on $\mathbb{C}^{n}$, see Section 3 below) and show that we can obtain the indirect boundary stabilization of this system, by adapting the method of Alabau developed in the context of coupled real wave equations with constant coefficients. We note here that the coupling coefficient a is considered as a function with $\|a\|_{L^{\infty}(\Omega)}$ sufficiently small. To obtain our result we need some geometric assumptions, two examples are presented to verify these assumptions.

Our paper is organized as follow: in Section 2, we shall present some notations and results on the Riemannian geometry in $\mathbb{R}^{n}$ generated by the principal part $\mathcal{A}$. In Section 3, we construct a suitable Riemannian metric on $\mathbb{C}^{n}$ and prove some formulas we need. In Section 4, we give some examples, where the geometric assumption is illustrated. In Section 5, we discuss briefly the well posedeness of the system (1)-(4). Section 6 is devoted to the proof of the indirect boundary stabilization result.

\section{Riemannian metric on $\mathbb{R}^{n}$ generated by the principal part $\mathcal{A}$}

\subsection{Notations}

Let $A(x)$ and $B(x)$ be, respectively, the coefficient matrix and its inverse:

$$
A(x)=\left(a_{i j}(x)\right)_{i, j=1, \ldots, n} \text { and } B(x)=\left(g_{i j}(x)\right)_{i, j=1, \ldots, n} .
$$

Let $\mathbb{R}^{n}$ have the usual topology and $x=\left(x_{1}, x_{2}, \ldots, x_{n}\right)$ be the natural coordinates system. For each $x \in \mathbb{R}^{n}$, we define the inner product on the tangent space $\mathbb{R}_{x}^{n}=\mathbb{R}^{n}$ setting, for all $X=\sum_{i=1}^{n} \alpha_{i} \frac{\partial}{\partial x_{i}}, Y=\sum_{i=1}^{n} \beta_{i} \frac{\partial}{\partial x_{i}}$

$$
g(X, Y)=\langle X, Y\rangle_{g}=\sum_{i, j=1}^{n} g_{i j}(x) \alpha_{i} \beta_{j} .
$$

The corresponding norm is

$$
\|X\|_{g}=\langle X, X\rangle_{g}^{1 / 2},
$$

and $\left(\mathbb{R}^{n}, g\right)$ becomes a Riemannian manifold with Riemannian metric $g$.

Remark 2.1. When $A=$ Identity, we find the usual dot product over $\mathbb{R}^{n}: X \cdot Y=$ $\sum_{i=1}^{n} \alpha_{i} \beta_{i}$, for all $X=\sum_{i=1}^{n} \alpha_{i} \frac{\partial}{\partial x_{i}}, Y=\sum_{i=1}^{n} \beta_{i} \frac{\partial}{\partial x_{i}}$ and the norm is defined by $|X|=$ $(X \cdot X)^{1 / 2}$. 
For a real valued function $f$ of $C^{1}(\Omega)$ and $X=\sum_{i=1}^{n} \alpha_{i}(x) \frac{\partial}{\partial x_{i}}$, we define the gradient of $f$ and the divergence of $X$ in the Euclidean metric by

$$
\operatorname{div}_{0}(X)=\sum_{i=1}^{n} \frac{\partial \alpha_{i}(x)}{\partial x_{i}}, \quad \nabla_{0} f=\sum_{i=1}^{n} \frac{\partial f}{\partial x_{i}} \frac{\partial}{\partial x_{i}} .
$$

We define the gradient $\nabla_{g} f$ of $f$ in the Riemannian metric $g$, via Riesz representation theorem, by

$$
X(f)=\left\langle\nabla_{g} f, X\right\rangle_{g},
$$

where $X$ is any vector field on the manifold $\left(\mathbb{R}^{n}, g\right)$. Denote the Levi-Civita connection in the Riemannian metric $g$ by $D$. The covariant differential $D h$ of a vector field $h=\sum_{i=1}^{n} h_{i}(x) \frac{\partial}{\partial x_{i}}$ is defined by

$$
D h(Y, X)=\left\langle D_{X} h, Y\right\rangle_{g},
$$

where $D_{X} h$ is the covariant derivative of $h$ with respect to $X$.

\subsection{Further relationships}

The following lemma provides further relationships (see [24]).

Lemma 2.2. Let $f, f_{1}, f_{2}$ be real valued functions of $C^{1}(\bar{\Omega})$ and $h=\sum_{i=1}^{n} h_{i}(x) \frac{\partial}{\partial x_{i}}$ a vector field on $\left(\mathbb{R}^{n}, g\right)$. Then

1.

2.

$$
h(f)=\left\langle\nabla_{g} f, h\right\rangle_{g}=\nabla_{0} f \cdot h .
$$

$$
\left\langle\nabla_{g} f_{1}, \nabla_{g} f_{2}\right\rangle_{g}=\nabla_{0} f_{1} \cdot A(x) \nabla_{0} f_{2} .
$$

3. A Green's formula. If $f_{1}, f_{2} \in H^{2}(\Omega)$ then

$$
\int_{\Omega} \mathcal{A} f_{1} f_{2}=\int_{\Omega}\left\langle\nabla_{g} f_{1}, \nabla_{g} f_{2}\right\rangle_{g}-\int_{\Gamma} \frac{\partial f_{1}}{\partial v_{\mathcal{A}}} f_{2} .
$$

4. An identity. If $f \in C^{1}(\bar{\Omega})$ then

$$
\left\langle\nabla_{g} f, \nabla_{g}(h(f))\right\rangle_{g}=D h\left(\nabla_{g} f, \nabla_{g} f\right)+\frac{1}{2} \operatorname{div}_{0}\left(h\left\|\nabla_{g} f\right\|_{g}^{2}\right)+\frac{1}{2}\left\|\nabla_{g} f\right\|_{g}^{2} \operatorname{div}_{0} h .
$$

\section{Riemannian metric on $\mathbb{C}^{n}$}

Using the inner product $\langle\cdot, \cdot\rangle_{g}$ on $\mathbb{R}^{n}$ we can define an inner product on $\mathbb{C}^{n}=\mathbb{C}_{x}^{n}$ (we take the same symbol $g$ ) by for all $Z_{1}, Z_{2} \in \mathbb{C}^{n}$

$$
\begin{aligned}
\left\langle Z_{1}, Z_{2}\right\rangle_{g}= & \left\langle\operatorname{Re} Z_{1}, \operatorname{Re} Z_{2}\right\rangle_{g}+\left\langle\operatorname{Im} Z_{1}, \operatorname{Im} Z_{2}\right\rangle_{g} \\
& -i\left(\left\langle\operatorname{Re} Z_{1}, \operatorname{Im} Z_{2}\right\rangle_{g}-\left\langle\operatorname{Im} Z_{1}, \operatorname{Re} Z_{2}\right\rangle_{g}\right),
\end{aligned}
$$


so the norm is

$$
\|Z\|_{g}^{2}=\langle Z, Z\rangle_{g}=\|\operatorname{Re} Z\|_{g}^{2}+\|\operatorname{Im} Z\|_{g}^{2} \text { for all } Z \in \mathbb{C}^{n} .
$$

Let $f$ be a complex valued function and $h$ be a vector field on $\left(\mathbb{R}^{n}, g\right)$. We put

$$
h(f):=h(\operatorname{Re} f)+i h(\operatorname{Im} f) \quad \text { and } \quad \nabla_{g} f:=\nabla_{g} \operatorname{Re} f+i \nabla_{g} \operatorname{Im} f .
$$

\section{Main formulas}

We give the counterpart of the Green's formula and the identity (5) for complex valued functions:

Lemma 3.1. 1. Let $f_{1}, f_{2}$ be complex valued functions in $H^{2}(\Omega)$. Then

$$
\operatorname{Re} \int_{\Omega} \mathcal{A} f_{1} \overline{f_{2}}=\operatorname{Re} \int_{\Omega}\left\langle\nabla_{g} f_{1}, \nabla_{g} f_{2}\right\rangle_{g}-\operatorname{Re} \int_{\Gamma} \frac{\partial f_{1}}{\partial v_{\mathcal{A}}} \overline{f_{2}} .
$$

2. Let $f$ be a complex valued function in $C^{1}(\bar{\Omega})$ and $h$ a vector field on $\left(\mathbb{R}^{n}, g\right)$. Then

$$
\begin{aligned}
\operatorname{Re}\left\langle\nabla_{g} f, \nabla_{g}(h(f))\right\rangle_{g}= & \operatorname{Dh}\left(\nabla_{g} \operatorname{Re} f, \nabla_{g} \operatorname{Re} f\right)+\operatorname{Dh}\left(\nabla_{g} \operatorname{Im} f, \nabla_{g} \operatorname{Im} f\right) \\
& +\frac{1}{2} \operatorname{div}_{0}\left(h\left\|\nabla_{g} f\right\|_{g}^{2}\right)-\frac{1}{2}\left\|\nabla_{g} f\right\|_{g}^{2} \operatorname{div}_{0} h .
\end{aligned}
$$

Proof. $\quad$ 1. Using Green's formula we obtain

$$
\begin{aligned}
\operatorname{Re} \int_{\Omega} \mathcal{A} f_{1} \overline{f_{2}}= & \operatorname{Re} \int_{\Omega} \mathcal{A}\left(\operatorname{Re} f_{1}+i \operatorname{Im} f_{1}\right)\left(\operatorname{Re} f_{2}-i \operatorname{Im} f_{2}\right) \\
= & \int_{\Omega} \mathcal{A} \operatorname{Re} f_{1} \operatorname{Re} f_{2}+\int_{\Omega} \mathcal{A} \operatorname{Im} f_{1} \operatorname{Im} f_{2} \\
= & \int_{\Omega}\left\langle\nabla_{g} \operatorname{Re} f_{1}, \nabla_{g} \operatorname{Re} f_{2}\right\rangle_{g}+\int_{\Omega}\left\langle\nabla_{g} \operatorname{Im} f_{1}, \nabla_{g} \operatorname{Im} f_{2}\right\rangle_{g} \\
& -\left(\int_{\Gamma} \frac{\partial \operatorname{Re} f_{1}}{\partial v_{\mathcal{A}}} \operatorname{Re} f_{2}-\int_{\Gamma} \frac{\partial \operatorname{Im} f_{1}}{\partial v_{\mathcal{A}}} \overline{\operatorname{Im}} \overline{f_{2}}\right) .
\end{aligned}
$$

The proof of desired formula is complete.

2. It is sufficient to observe that

$\operatorname{Re}\left\langle\nabla_{g} f, \nabla_{g}(h(f))\right\rangle_{g}=\left\langle\nabla_{g} \operatorname{Re} f, \nabla_{g}(h(\operatorname{Re} f))\right\rangle_{g}+\left\langle\nabla_{g} \operatorname{Im} f, \nabla_{g}(h(\operatorname{Im} f))\right\rangle_{g}$,

so, (6) follows from (5).

Remark 3.2. We can see that there exist two positive constants $\alpha$ and $\beta$ such that for all $f$ of $H^{1}(\Omega)$ we have $\alpha \int_{\Omega}\left|\nabla_{0} f\right|^{2} \leq \int_{\Omega}\left\|\nabla_{g} f\right\|_{g}^{2}=\int_{\Omega}\left\|\operatorname{Re} \nabla_{g} f\right\|_{g}^{2}+$ $\int_{\Omega}\left\|\operatorname{Im} \nabla_{g} f\right\|_{g}^{2} \leq \beta \int_{\Omega}\left|\nabla_{0} f\right|^{2}$.

In all this paper, $C_{1}$ and $C_{2}$ are the positive constants such that $\int_{\Omega}|f|^{2} \leq$ $C_{1}^{2} \int_{\Omega}\left\|\nabla_{g} f\right\|_{g}^{2}$ and $\int_{\Gamma_{1}}|f|^{2} \leq C_{2}^{2} \int_{\Omega}\left\|\nabla_{g} f\right\|_{g}^{2}$ for all functions $f$ of $H_{\Gamma_{0}}^{1}(\Omega) . C$ is a generic positive constant which does not depend on the initial data. 


\section{Geometric assumptions and examples}

Assume that there exists a real vector field $h \in\left[C^{1}(\bar{\Omega})\right]^{n}$ on the Riemannian manifold $\left(\mathbb{R}^{n}, g\right)$, a constant $m_{0}>0$ such that

$$
D h(X, X) \geq m_{0}\|X\|_{g}^{2}, \quad \text { for all } \quad X \in \mathbb{R}_{x}^{n},
$$

and

$$
2 m_{0}>C_{1} C_{h}
$$

where $C_{h}=\sup _{x \in \Omega}\left\|\nabla_{g}\left(\operatorname{div}_{0} h\right)\right\|_{g}$

We assume that $\Gamma_{0}, \Gamma_{1}$ are taken as

$$
\Gamma_{0}=\{x \in \Gamma \mid h \cdot v \leq 0\} \quad \text { and } \quad \Gamma_{1}=\left\{x \in \Gamma \mid h \cdot v \geq h_{0}>0\right\} .
$$

We now give two examples that verify assumptions (7) and (8).

Example 4.1. If $\left(a_{i j}\right)$ is a positive, symmetric constant matrix, then we may take $h=\sum_{i=1}^{n}\left(x_{i}-x_{i}^{0}\right) \frac{\partial}{\partial x_{i}}, x_{0} \in \mathbb{R}^{n}$ to satisfy (7) (see [24]). Moreover, in this case the condition (8) holds true as well: $\operatorname{div}_{0} h=\sum_{i=1}^{n} \frac{\partial}{\partial x_{i}}\left(x_{i}-x_{i}^{0}\right)=n$, so $C_{h}=0$.

Example 4.2. In [11] the authors have proved that the geometric condition (7), derived by Yao in term of the Riemannian geometry method, is equivalent to the following analytical condition given by Wyler [23] for boundary stabilization of wave equations with variable coefficients:

$$
\left\{\begin{array}{l}
\left(p_{i j}\right) \text { is a uniformly positive definite matrix in } \Omega, \text { where } \\
p_{i j}=p_{j i}=\sum_{k=1}^{k=n} a_{i k} \frac{\partial h_{j}}{\partial x_{k}}+\sum_{k=1}^{k=n} a_{j k} \frac{\partial h_{i}}{\partial x_{k}}-\nabla_{0} a_{i j} \cdot h .
\end{array}\right.
$$

If $h(x)=\sum_{i=1}^{n} h_{i}(x) \frac{\partial}{\partial x_{i}}$ is a vector field in $\mathbb{R}^{n}$ such that $\operatorname{div}_{0} h$ is a constant (8) is verified. If $\left(a_{i j}\right)$ is a matrix defined by

$$
a_{i j}\left(x_{1}, x_{2}, \ldots, x_{n}\right)= \begin{cases}0 & i \neq j, \\ f_{i}\left(x_{i}\right) & i=j\end{cases}
$$

where, for all $i=1, \ldots, n, f_{i}: \mathbb{R} \rightarrow \mathbb{R}$ is a function of class $C^{1}$ satisfying the condition

$$
\min f_{i}>0
$$

and

$$
\begin{aligned}
\min \left(2 f_{i} \frac{\partial h_{i}}{\partial x_{i}}-\frac{\partial f_{i}}{\partial x_{i}} h_{i}\right) & >0, \quad i=j, \\
f_{i} \frac{\partial h_{j}}{\partial x_{i}}+f_{j} \frac{\partial h_{i}}{\partial x_{j}} & =0, \quad i \neq j,
\end{aligned}
$$

$\left(p_{i j}\right)$ is uniformly positive definite matrix in $\Omega$.

As an example of such vector and matrix we can take $h_{i}=x_{i}-x_{i}^{0}, x_{0} \in \mathbb{R}^{n}$ and $f_{i}\left(x_{i}\right)=\left(x_{i}-x_{i}^{0}\right)^{2}+\beta$ where $\beta \in \mathbb{R}_{+}^{*}$. 
Remark 4.1. We note that assumption (8) has been used in [14] to study the exact controllability of real wave equation with constant coefficients.

\section{Existence and regularity of solutions}

Let $\mathbb{A}: D(\mathbb{A}) \subset H_{\Gamma_{0}}^{1}(\Omega) \times H_{0}^{1}(\Omega) \rightarrow H_{\Gamma_{0}}^{1}(\Omega) \times H_{0}^{1}(\Omega)$ be the operator defined by

$$
\mathbb{A}\left(u_{1}, u_{2}\right)=\left(\mathfrak{i} \mathcal{A} u_{1}+\mathfrak{i} a u_{2}, \mathfrak{i} \mathcal{A} u_{2}+\mathfrak{i} a u_{1}\right),
$$

where

$$
D=D(\mathbb{A})=\left\{\begin{array}{c}
\left(u_{1}, u_{2}\right) \in H_{\Gamma_{0}}^{1}(\Omega) \times H_{0}^{1}(\Omega) \mid\left(\mathcal{A} u_{1}, \mathcal{A} u_{2}\right) \in H_{\Gamma_{0}}^{1}(\Omega) \times H_{0}^{1}(\Omega) \\
\text { and } \frac{\partial u_{1}}{\partial v_{\mathcal{A}}}+\left.\mathfrak{i} b \mathcal{A} u_{1}\right|_{\Gamma_{1}}=0
\end{array}\right\} .
$$

Using the idea in [9] we can show that (1)-(4) is equivalent to

$$
\left\{\begin{array}{l}
(y, z)_{t}=\mathbb{A}(y, z) \\
(y(0), z(0))=\left(y_{0}, z_{0}\right) .
\end{array}\right.
$$

Then we have the following result:

Theorem 5.1. Let $N \geq 1$, if $\left(y_{0}, z_{0}\right) \in D\left(\mathbb{A}^{N}\right)$ the system (1)-(4) has a unique solution $(y, z) \in C^{N-j}\left([0,+\infty) ; D\left(\mathbb{A}^{j}\right)\right)$ for $j=0, \ldots, N$. Here $D\left(\mathbb{A}^{1}\right)=D(\mathbb{A})$ and $D\left(\mathbb{A}^{N}\right)=\left\{\left(u_{1}, u_{2}\right) \in D\left(\mathbb{A}^{N-1}\right) \mid \mathbb{A}\left(u_{1}, u_{2}\right) \in D\left(\mathbb{A}^{N-1}\right)\right\}$ for $N \geq 2$.

Proof. It is sufficient to prove that $(-\mathbb{A})$ is a maximal monotone operator in the space $H_{\Gamma_{0}}^{1}(\Omega) \times H_{0}^{1}(\Omega)$ (see Theorem VII4 and Theorem VII5 in [8]).

First, we must show that given $\left(v_{1}, v_{2}\right) \in H_{\Gamma_{0}}^{1}(\Omega) \times H_{0}^{1}(\Omega)$, there exists a solution $\left(u_{1}, u_{2}\right) \in D(\mathbb{A})$ such that $[I-\mathbb{A}]\left(u_{1}, u_{2}\right)=\left(v_{1}, v_{2}\right)$. This yields a corresponding elliptic problem

$$
\begin{aligned}
& u_{1}-i \mathcal{A} u_{1}-i a u_{2}=v_{1} \text { in } \Omega, \\
& u_{2}-i \mathcal{A} u_{2}-i a u_{1}=v_{2} \text { in } \Omega, \\
& u_{1}=0 \text { on } \Gamma_{0} \text { and } u_{2}=0 \text { on } \Gamma, \\
& \frac{\partial u_{1}}{\partial v}+b\left(u_{1}-v_{1}\right)=0 \text { on } \Gamma_{1},
\end{aligned}
$$

and elliptic theory gives the desired conclusion. To prove that $(-\mathbb{A})$ is a monotone operator in $H_{\Gamma_{0}}^{1}(\Omega) \times H_{0}^{1}(\Omega)$, we have some difficulty to do it directly. To overcome this difficulty we use some results in semigroup theory: First, we can easily obtain, making use of the Faedo-Galerkin method (see $[4,5,10,16]$ ), that if $\left(y_{0}, z_{0}\right) \in H_{\Gamma_{0}}^{1}(\Omega) \times H_{0}^{1}(\Omega)$ the system (1)-(4) has a unique solution $(y, z) \in$ $C\left([0,+\infty) ; H_{\Gamma_{0}}^{1}(\Omega) \times H_{0}^{1}(\Omega)\right)$ and that if $\left(y_{0}, z_{0}\right) \in D$ the system (1)-(4) has a unique solution $(y, z) \in C([0,+\infty) ; D) \cap C^{1}\left([0,+\infty) ; H_{\Gamma_{0}}^{1}(\Omega) \times H_{0}^{1}(\Omega)\right)$. For all $t \geq 0$, we consider the mapping

$$
T(t): H_{\Gamma_{0}}^{1}(\Omega) \times H_{0}^{1}(\Omega) \rightarrow H_{\Gamma_{0}}^{1}(\Omega) \times H_{0}^{1}(\Omega)
$$

defined by

$$
T(t)\left(y_{0}, z_{0}\right)=(y(t), z(t)),
$$

where $(y, z)$ is the solution of $(P)$ (or (1)-(4)). 
It is clear that $(T(t))_{t \geq 0}$ is a $C_{0}$-semigroup on $H_{\Gamma_{0}}^{1}(\Omega) \times H_{0}^{1}(\Omega)$ and (see Lemma 6.1 below)

$$
\begin{aligned}
\left\|T(t)\left(y_{0}, z_{0}\right)\right\|_{H_{\Gamma_{0}}^{1}(\Omega) \times H_{0}^{1}(\Omega)}^{2} & =\|(y, z)\|_{H_{\Gamma_{0}}^{1}(\Omega) \times H_{0}^{1}(\Omega)}^{2}=2 E(t) \\
& \leq 2 E(0)=\left\|\left(y_{0}, z_{0}\right)\right\|_{H_{\Gamma_{0}}^{1}(\Omega) \times H_{0}^{1}(\Omega)}^{2} .
\end{aligned}
$$

Then $(T(t))_{t \geq 0}$ is a $C_{0}$-semigroup of contraction.

Denote by $\mathbb{B}$ the infinitesimal generator of $(T(t))_{t>0}$. By Theorem 8 , Chapter 3 in $[10], \mathbb{B}$ is a maximal monotone operator in $H_{\Gamma_{0}}^{1}(\Omega) \times H_{0}^{1}(\Omega)$.

Let $\left(y_{0}, z_{0}\right) \in D(\mathbb{A})$ then

$$
\frac{d}{d t} T(t)\left(y_{0}, z_{0}\right)=(y, z)_{t}=\mathbb{A}(y, z)=\mathbb{A} T(t)\left(y_{0}, z_{0}\right),
$$

which implies that $\left[\frac{d}{d t} T(t)\left(y_{0}, z_{0}\right)\right]_{t=0}=\mathbb{A}\left(y_{0}, z_{0}\right)$ and therefore $\mathbb{A} \subset \mathbb{B}$. This implies that $\mathbb{A}$ is a monotone operator in $H_{\Gamma_{0}}^{1}(\Omega) \times H_{0}^{1}(\Omega)$.

\section{Indirect boundary stabilization result}

Consider the total energy $E$ of (1)-(4) defined by

$$
E(t)=E(y(t), z(t))=E_{1}(y(t))+E_{2}(z(t))+\operatorname{Re} \int_{\Omega} a y \bar{z},
$$

where

$$
E_{1}(t)=E_{1}(y(t))=\frac{1}{2}\left(\int_{\Omega}\left\|\nabla_{g} y\right\|_{g}^{2}\right) \quad \text { and } \quad E_{2}(t)=E_{2}(z(t))=\frac{1}{2}\left(\int_{\Omega}\left\|\nabla_{g} z\right\|_{g}^{2}\right) .
$$

We can see that $E$ is equivalent to $E_{1}+E_{2}$ when we take $\|a\|_{L^{\infty}(\Omega)}$ sufficiently small. lemma:

The dissipative property of the solution of (1)-(4) is given by the following

Lemma 6.1. We have for all $t \geq 0, E^{\prime}(t)=-\int_{\Gamma_{1}} b\left|y_{t}\right|^{2} \leq 0$.

Proof. We multiply both side (1) by $\bar{y}_{t}$, integrate over $\Omega$, take the real part, use Lemma 3.1, and finally use the boundary condition (3), to find

$$
\operatorname{Re} \int_{\Omega}\left\langle\nabla_{g} y, \nabla_{g} y_{t}\right\rangle_{g}+\int_{\Gamma_{1}} b\left|y_{t}\right|^{2}+\operatorname{Re} \int_{\Omega} a z \overline{y_{t}}=0 .
$$

We obtain a similar identity for $z$

$$
\operatorname{Re} \int_{\Omega}\left\langle\nabla_{g} z, \nabla_{g} z_{t}\right\rangle_{g}+\operatorname{Re} \int_{\Omega} a y \overline{z_{t}}=0 .
$$

But

$$
E^{\prime}(t)=\operatorname{Re} \int_{\Omega}\left\langle\nabla_{g} y, \nabla_{g} y_{t}\right\rangle_{g}+\operatorname{Re} \int_{\Omega}\left\langle\nabla_{g} z, \nabla_{g} z_{t}\right\rangle_{g}+\operatorname{Re} \int_{\Omega} a(y \bar{z})_{t},
$$

and we find the result. 
Remark 6.2. We deduce from Lemma 6.1 that

$$
E(T) \leq E(0)
$$

and

$$
\int_{\Sigma_{1}}\left|y_{t}\right|^{2} \leq C E(0)
$$

Our main result is

Theorem 6.3. Let $N \geq 1$. For any initial data $\left(y_{0}, z_{0}\right) \in D\left(A^{N}\right)$, the energy $E$ of the solution of system (1)-(4) decays polynomially:

$$
E(y(t), z(t)) \leq \frac{C}{t^{N}} \sum_{p=0}^{p=N} E\left(y^{(p)}(0), z^{(p)}(0)\right) \quad \text { for all } t>0 .
$$

Proof. To prove our result we estimate $\int_{0}^{T} E_{1}(t)$ and $\int_{0}^{T} E_{2}(t)$ then, after summing up these two estimates, we conclude applying the Theorem 3.1 in [2] with $K=1$.

Step 1. We prove an estimate useful to estimate the term $\int_{0}^{T} E_{1}(t)$.

For fixed $t$, we consider $w$ the solution of the problem

$$
\mathcal{A} w=0 \quad \text { in } \Omega, \quad w=y \text { on } \Gamma .
$$

Using elliptic regularity (Lemma 2.1 in [6]), we can see that

$$
\int_{\Omega}|w|^{2} \leq C \int_{\Gamma_{1}}|y|^{2} \leq C E_{1}(t) .
$$

If we use this inequality with the derivatives, integrate over $[0, T]$ and use (10) we obtain

$$
\int_{Q}\left|w_{t}\right|^{2} \leq C \int_{\Sigma_{1}}\left|y_{t}\right|^{2} \leq C E(0) .
$$

On the other hand, we have

$$
\operatorname{Re} \int_{\Omega} \mathcal{A} w \bar{z}=0
$$

then

$$
\operatorname{Re} \int_{\Omega}\left\langle\nabla_{g} w, \nabla_{g} z\right\rangle_{g}=0 .
$$

Multiplying the conjugate of (2) by $y-w$, integrating over $Q$ and taking the real part

$$
\operatorname{Im} \int_{Q} \overline{z_{t}}(y-w)+\operatorname{Re} \int_{Q} \mathcal{A} \bar{z}(y-w)+\int_{Q} a|y|^{2}-\operatorname{Re} \int_{Q} a \bar{y} w=0,
$$

then, by Lemma 3.1, (12) and the integration by parts, we find

$$
\begin{aligned}
\left.\operatorname{Im} \int_{\Omega} \bar{z}(y-w)\right|_{0} ^{T}-\operatorname{Im} \int_{Q} & \bar{z}\left(y_{t}-w_{t}\right) \\
& +\operatorname{Re} \int_{Q}\left\langle\nabla_{g} z, \nabla_{g} y\right\rangle_{g}+\int_{Q} a|y|^{2}-\operatorname{Re} \int_{Q} a \bar{y} w=0 .
\end{aligned}
$$


If we multiply (1) by $(-\bar{z})$, integrate over $Q$, take the real part and sum the result with the last identity, we find

$$
\int_{Q} a|z|^{2}=\left.\operatorname{Im} \int_{\Omega} \bar{z}(y-w)\right|_{0} ^{T}+\operatorname{Im} \int_{Q} \bar{z} w_{t}+\int_{Q} a|y|^{2}-\operatorname{Re} \int_{Q} a \bar{y} w .
$$

But

$$
\left|\int_{\Omega} \bar{z}(y-w)\right| \leq C\left(\int_{\Omega}|y|^{2}+\int_{\Omega}|z|^{2}+\int_{\Omega}|w|^{2}\right) \leq C E(0),
$$

then, for all $\varepsilon>0$, we have

$$
\int_{Q} a|z|^{2} \leq C E(0)+\frac{\varepsilon}{a_{*} 2} \int_{Q} a|z|^{2}+\frac{1}{2 \varepsilon} \int_{Q}\left|w_{t}\right|^{2}+C\|a\|_{L^{\infty}(\Omega)} \int_{0}^{T} E_{1}(t) .
$$

Using (11) and choosing $\varepsilon=a_{*}$ we find

$$
\int_{Q} a|z|^{2} \leq C E(0)+C\|a\|_{L^{\infty}(\Omega)} \int_{0}^{T} E_{1}(t) .
$$

Step 2. An estimate of the term $\int_{0}^{T} E_{1}(t)$.

Multiplying (1) by $2 h(\bar{y})+\operatorname{div}_{0} h \bar{y}$, integrating over $Q$ and taking the real part, we obtain

$$
\begin{aligned}
0= & \operatorname{Im} \int_{Q} y_{t}\left(2 h(\bar{y})+\operatorname{div}_{0} h \bar{y}\right)-\operatorname{Re} \int_{Q} \mathcal{A} y\left(2 h(\bar{y})+\operatorname{div}_{0} h \bar{y}\right) \\
& -\operatorname{Re} \int_{Q} a z\left(2 h(\bar{y})+\operatorname{div}_{0} h \bar{y}\right) .
\end{aligned}
$$

By integration by parts, we have

$$
\int_{Q} y_{t} h(\bar{y})=\left.\int_{\Omega} y h(\bar{y})\right|_{0} ^{T}-\int_{Q} y h\left(\overline{y_{t}}\right) .
$$

We invoke the standard divergence identity

to obtain

$$
\int_{\Omega} s \cdot \nabla_{0} \psi=\int_{\Gamma} \psi s \cdot v-\int_{\Omega} \psi \operatorname{div}_{0} s,
$$

$$
\begin{aligned}
\int_{Q} y_{t} h(\bar{y}) & =\left.\int_{\Omega} y h(\bar{y})\right|_{0} ^{T}-\int_{\Sigma} h \cdot v y \overline{y_{t}}+\int_{Q} \overline{y_{t}} \operatorname{div}_{0}(h y) \\
& =\left.\int_{\Omega} y h(\bar{y})\right|_{0} ^{T}-\int_{\Sigma} h \cdot v y \overline{y_{t}}+\int_{Q} \overline{y_{t}} \operatorname{div}_{0} h y+\int_{Q} \overline{y_{t}} h(y) .
\end{aligned}
$$

Then

$$
2 \operatorname{Im} \int_{Q} y_{t} h(\bar{y})=\left.\operatorname{Im} \int_{\Omega} y h(\bar{y})\right|_{0} ^{T}-\operatorname{Im} \int_{\Sigma} h \cdot v y \overline{y_{t}}-\operatorname{Im} \int_{Q} y_{t} \operatorname{div}_{0} h \bar{y},
$$

SO

$$
\operatorname{Im} \int_{Q} y_{t}\left(2 h(\bar{y})+\operatorname{div}_{0} h \bar{y}\right)=\left.\operatorname{Im} \int_{\Omega} y h(\bar{y})\right|_{0} ^{T}-\operatorname{Im} \int_{\Sigma_{1}} h \cdot v y \overline{y_{t}} .
$$


If we use Lemma 3.1, we find

$$
\begin{aligned}
\operatorname{Re} \int_{Q} \mathcal{A} y\left(2 h(\bar{y})+\operatorname{div}_{0} h \bar{y}\right) \\
=-\operatorname{Re} \int_{\Sigma} \frac{\partial y}{\partial v_{\mathcal{A}}}\left(2 h(\bar{y})+\operatorname{div}_{0} h \bar{y}\right) \\
\quad+2 \int_{Q} \operatorname{Re}\left\langle\nabla_{g} y, \nabla_{g}(h(y))\right\rangle_{g}+\int_{Q} \operatorname{Re}\left\langle\nabla_{g} y, \nabla_{g}\left(\operatorname{div}_{0} h y\right)\right\rangle_{g} .
\end{aligned}
$$

Indeed, from the identity (6) we obtain

$$
\begin{aligned}
\operatorname{Re} \int_{Q} \mathcal{A} y & \left(2 h(\bar{y})+\operatorname{div}_{0} h \bar{y}\right) \\
= & -\operatorname{Re} \int_{\Sigma} \frac{\partial y}{\partial v_{\mathcal{A}}}\left(2 h(\bar{y})+\operatorname{div}_{0} h \bar{y}\right)+2 \int_{Q} D h\left(\nabla_{g} \operatorname{Re} y, \nabla_{g} \operatorname{Re} y\right) \\
& +2 \int_{Q} D h\left(\nabla_{g} \operatorname{Im} y, \nabla_{g} \operatorname{Im} y\right)+\int_{Q} h \cdot \nabla_{0}\left(\left\|\nabla_{g} y\right\|_{g}^{2}\right) \\
& +\operatorname{Re} \int_{Q}\left\langle\nabla_{g} y, \nabla_{g}\left(\operatorname{div}_{0} h\right)\right\rangle \bar{y}+\int_{Q} \operatorname{div}_{0} h\left\|\nabla_{g} y\right\|_{g}^{2} .
\end{aligned}
$$

Using the divergence identity, we find

$$
\begin{aligned}
\operatorname{Re} \int_{Q} \mathcal{A} y & \left(2 h(\bar{y})+\operatorname{div}_{0} h \bar{y}\right) \\
= & -\operatorname{Re} \int_{\Sigma} \frac{\partial y}{\partial v_{\mathcal{A}}}\left(2 h(\bar{y})+\operatorname{div}_{0} h \bar{y}\right) \\
& +2 \int_{Q} D h\left(\nabla_{g} \operatorname{Re} y, \nabla_{g} \operatorname{Re} y\right)+2 \int_{Q} D h\left(\nabla_{g} \operatorname{Im} y, \nabla_{g} \operatorname{Im} y\right) \\
& +\int_{\Sigma} h \cdot v\left\|\nabla_{g} y\right\|_{g}^{2}+\operatorname{Re} \int_{Q}\left\langle\nabla_{g} y, \nabla_{g}\left(\operatorname{div}_{0} h\right)\right\rangle_{g} \bar{y} .
\end{aligned}
$$

Since, $\operatorname{Re} y=\operatorname{Im} y=0$ on $\Gamma_{0}$, then we have (see [24])

$$
h(\operatorname{Re} y)=\frac{h \cdot v}{\left\|v_{\mathcal{A}}(x)\right\|_{g}^{2}} \frac{\partial \operatorname{Re} y}{\partial v_{\mathcal{A}}} \quad \text { and } \quad\left\|\nabla_{g} \operatorname{Re} y\right\|_{g}^{2}=\frac{1}{\left\|v_{\mathcal{A}}(x)\right\|_{g}^{2}}\left(\frac{\partial \operatorname{Re} y}{\partial v_{\mathcal{A}}}\right)^{2}
$$

and

$$
h(\operatorname{Im} y)=\frac{h \cdot v}{\left\|v_{\mathcal{A}}(x)\right\|_{g}^{2}} \frac{\partial \operatorname{Im} y}{\partial v_{\mathcal{A}}} \quad \text { and } \quad\left\|\nabla_{g} \operatorname{Im} y\right\|_{g}^{2}=\frac{1}{\left\|v_{\mathcal{A}}(x)\right\|_{g}^{2}}\left(\frac{\partial \operatorname{Im} y}{\partial v_{\mathcal{A}}}\right)^{2} .
$$

So

$$
h(y)=\frac{h \cdot v}{\left\|v_{\mathcal{A}}(x)\right\|_{g}^{2}} \frac{\partial y}{\partial v_{\mathcal{A}}} \quad \text { and } \quad\left\|\nabla_{g} y\right\|_{g}^{2}=\frac{1}{\left\|v_{\mathcal{A}}(x)\right\|_{g}^{2}}\left|\frac{\partial y}{\partial v_{\mathcal{A}}}\right|^{2}
$$


Then

$$
\begin{aligned}
-\operatorname{Re} \int_{Q} \mathcal{A} y & \left(2 h(\bar{y})+\operatorname{div}_{0} h \bar{y}\right) \\
= & \int_{\Sigma_{0}} \frac{h \cdot v}{\left\|v_{\mathcal{A}}(x)\right\|_{g}^{2}}\left|\frac{\partial y}{\partial v_{\mathcal{A}}}\right|^{2}-\int_{\Sigma_{1}} h \cdot v\left\|\nabla_{g} y\right\|_{g}^{2} \\
& +\operatorname{Re} \int_{\Sigma_{1}} \frac{\partial y}{\partial v_{\mathcal{A}}}\left(2 h(\bar{y})+\operatorname{div}_{0} h \bar{y}\right)-2 \int_{Q} D h\left(\nabla_{g} \operatorname{Re} y, \nabla_{g} \operatorname{Re} y\right) \\
& -2 \int_{Q} D h\left(\nabla_{g} \operatorname{Im} y, \nabla_{g} \operatorname{Im} y\right)-\operatorname{Re} \int_{Q}\left\langle\nabla_{g} y, \nabla_{g}\left(\operatorname{div}_{0} h\right)\right\rangle_{g} \bar{y}
\end{aligned}
$$

Finally, we insert (15) and (16) in (14) to obtain

$$
\begin{aligned}
2\left(\int_{Q} \operatorname{Dh}\left(\nabla_{g} \operatorname{Re} y, \nabla_{g} \operatorname{Re} y\right)+\int_{Q} \operatorname{Dh}\left(\nabla_{g} \operatorname{Im} y, \nabla_{g} \operatorname{Im} y\right)\right) & \\
& =I_{\Omega}+I_{\Sigma_{0}}+I_{\Sigma_{1}}+I_{Q}
\end{aligned}
$$

where

$$
\begin{aligned}
I_{\Omega} & =\left.\operatorname{Im} \int_{\Omega} y h(\bar{y})\right|_{0} ^{T}, \\
I_{\Sigma_{0}} & =\int_{\Sigma_{0}} \frac{h \cdot v}{\left\|v_{\mathcal{A}}(x)\right\|_{g}^{2}}\left|\frac{\partial y}{\partial v_{\mathcal{A}}}\right|^{2}, \\
I_{\Sigma_{1}} & =-\operatorname{Im} \int_{\Sigma_{1}} h \cdot v y \overline{y_{t}}-\int_{\Sigma_{1}} h \cdot v\left\|\nabla_{g} y\right\|_{g}^{2}-\operatorname{Re} \int_{\Sigma_{1}} b y_{t}\left(2 h(\bar{y})+\operatorname{div}_{0} h \bar{y}\right), \\
I_{Q} & =-\operatorname{Re} \int_{Q}\left\langle\nabla_{g} y, \nabla_{g}\left(\operatorname{div}_{0} h\right)\right\rangle_{g} \bar{y}-\operatorname{Re} \int_{Q} a z\left(2 h(\bar{y})+\operatorname{div}_{0} h \bar{y}\right) .
\end{aligned}
$$

We can see that by (9)

$$
I_{\Omega} \leq C E(0)
$$

We have

$$
I_{\Sigma_{0}} \leq 0
$$

We have for all $\varepsilon>0$

$$
\begin{gathered}
I_{\Sigma_{1}} \leq \frac{C}{\varepsilon} \int_{\Sigma_{1}}\left|y_{t}\right|^{2}+C \varepsilon \int_{Q}\left\|\nabla_{g} y\right\|_{g}^{2}+\left(C \varepsilon-h_{0}\right) \int_{\Sigma_{1}}\left\|\nabla_{g} y\right\|_{g}^{2} \\
\quad \stackrel{(10)}{\leq} C E(0)+C \varepsilon \int_{0}^{T} E_{1}(t)+\left(C \varepsilon-h_{0}\right) \int_{\Sigma_{1}}\left\|\nabla_{g} y\right\|_{g}^{2} .
\end{gathered}
$$

We also have, for all $\eta>0$, by (13)

$$
I_{Q} \leq C E(0)+\left(\eta C_{h}^{2}+\frac{C_{1}^{2}}{\eta}\right) \int_{0}^{T} E_{1}(t)+C\|a\|_{L^{\infty}(\Omega)} \int_{0}^{T} E_{1}(t) .
$$


Inserting the above estimates for $I_{\Omega}, I_{\Sigma_{l}}(l=0,1)$ and $I_{Q}$ in (17), using (7) and (8), and choosing $\eta=\frac{C_{1}}{C_{h}}, \varepsilon$ and $\|a\|_{L^{\infty}(\Omega)}$ sufficiently small, we find

$$
\int_{0}^{T} E_{1}(t) \leq C E(0) .
$$

Step 3. An estimate of the term $\int_{0}^{T} E_{2}(t)$.

First we have, by (13) and (18),

$$
\int_{Q}|z|^{2} \leq C E(0)=E(y(0), z(0)) .
$$

If we use this inequality with the derivatives, we obtain

$$
\int_{Q}\left|z_{t}\right|^{2} \leq C E\left(y_{t}(0), z_{t}(0)\right) .
$$

On the other hand, if we multiply (2) by $\bar{z}$, integrate over $Q$, take the real part and we use Lemma 3.1, we find

$$
\int_{Q}\left\|\nabla_{g} z\right\|_{g}^{2}=\operatorname{Im} \int_{Q} z_{t} \bar{z}-\operatorname{Re} \int_{Q} a y \bar{z} .
$$

If we use Cauchy Schwarz, (18), (19) and (20) we find

$$
\int_{0}^{T} E_{2}(t) \leq C\left(E(y(0), z(0))+E\left(y_{t}(0), z_{t}(0)\right)\right) .
$$

Step 4. We can now conclude the result of Theorem 6.3. We have, for all $T>0$

$$
\begin{aligned}
\int_{0}^{T} E(y(t), z(t)) & =\int_{0}^{T} E_{1}(y(t))+\int_{0}^{T} E_{2}(z(t))+\operatorname{Re} \int_{Q} a y \bar{z} \\
& \leq C\left(E(y(0), z(0))+E\left(y_{t}(0), z_{t}(0)\right)\right) .
\end{aligned}
$$

The desired conclusion follows from Theorem 3.1 in [2] with $K=1$.

\section{Acknowledgements}

The authors would like to thank the referee for giving us constructive remarks which resulted in this final version of our work and they would like to thank professor Pierre Rouchon (Ecole des Mines de Paris, Centre Automatique et Systèmes) for the related physical model. 


\section{References}

[1] A. Aassila, Strong asymptotic stability of a compactly coupled system of wave equations. App. Math. Letters 14 (2001), 285-290.

[2] F. Alabau, Indirect boundary stabilization of weakly coupled hyperbolic systems. SIAM J. Control Optimization 41 (2002), 511-541.

[3] F. Alabau, P. Cannarsa and V. Komornick, Indirect internal stabilization of weakly coupled evolution equations. J. of Evolution Equation 2 (2002), 127-150.

[4] D. Andrade and A. Mognon, Global solutions for a system of Klein-Gordon equations with memory. Bol. Soc. Paran. Mat. 21, 1/2 (2003), 127-138.

[5] R. F. Apolaya, H. R. Clark and A. J. Feitosa, On a nonlinear coupled system with internal damping. EJDE Journal 64 (2000), 1-17.

[6] C. Bernardi and Y. Maday, Approximations spectrales de problèmes aux limites elliptiques. Springer-Verlag, 1992.

[7] A. Beyrath, Indirect internal stabilization of coupled systems with locally distributed damping. C. R. Acad. Sci. 333, I (2001), 451-465.

[8] H. Brezis, Analyse fonctionnelle. Masson, 1987.

[9] R. Cipolatti, E. Machtyngier and E. Siqueira, Nonlinear boundary feedback stabilization for Schrödinger equations. Differential and integral equations journal 9 (1996), $1-15$.

[10] R. Dautray and J.L. Lions, Analyse mathématique et calcul numérique pour les sciences et les techniques. Masson, Paris, Tome 8, 1985.

[11] S. Feng and X. Feng, A note on geometric conditions for boundary control of wave equations with variable coefficients. J. Math. Anal. Appl. 271, 1 (2002), 59-65.

[12] S. Feng and X. Feng, Nonlinear internal damping of wave equations with variable coefficients. Acta Math. Sin. (Engl. Ser.) 20, 6 (2004), 1057-1072.

[13] I. Hamchi, Uniform decay rates for second-order hyperbolic equations with variable coefficients. Asymptotic Analysis journal 57, 1-2 (2008), 71-82.

[14] I. Lasiecka and R. Triggiani, Exact controllability of the wave equation with Neumann boundary control. App. Math. Optim. 19 (1989), 243-290.

[15] I. Lasiecka, R. Triggiani and P.F. Yao, Inverse/ observability estimates for second order hyperbolic equations with variables coefficients. Math. Ana. Appl. 235 (1999), $13-57$.

[16] J. L. Lions, Quelques méthodes de résolution des problèmes aux limites nonlinéaires. Dunod-Gauthier Villars, 1969.

[17] E. Machtyngier, Contrôlabilité exacte et stabilisation frontière de l'équation de Schrödinger. C. R. Acad. Sci. 310, 1 (1990), 801-806.

[18] E. Machtyngier and E. Zuazua, Stabilization of Schrödinger equation. Portugaliae Mathematica 51, 2 (1994), 243-256.

[19] S. Nicaise and C. Pignotti, Internal and boundary observability estimates for heterogeneous Maxwell's system. App., Math., Optim. 54 (2006), 47-70.

[20] P. Rouchon, Quantum systems and control. Article submitted to the proceedings of the conference in honor of Claude Lobry, Saint Louis (2007). 
[21] D. Russell, A general framework for the study of indirect damping mechanisms in elastic systems. Journal of Math. Ana. and Appl. 173 (1993), 339-358.

[22] C. Shugen and G. Yuxia, Boundary stabilization of wave equations with variable coefficients and memory. Differential Integral Equations 17, 5-6 (2004), 669-680.

[23] A. Wyler, Stability of wave equations with dissipative boundary conditions in a bounded domain. Differential Integral Equations 7 (1994), 345-366.

[24] P. F. Yao, On the observabillity inequalities for exact controllability of wave equations with variable coefficients. SIAM J. Control Optim. 37, 5 (1999), 1568-1599.

[25] P. F. Yao, Observability inequalities for the Euler-Bernoulli Plate with variable coefficients. Contemporary Mathematics 268 (2000), 383-406.

[26] P. F. Yao, Observabillity inequalities for Shalow Shells. SIAM J. Control Optim. 38 (2000), 1729-1756.

Ilhem Hamchi and Salah Eddine Rebiai

Department of Mathematics

University of Batna

05000, Algeria

e-mail: hamchi_ilhem@yahoo.fr

rebiai@hotmail.fr

Received: 1 December 2006.

Revised: 28 April 2008.

Accepted: 12 May 2008. 